\title{
Analysis of esophagogastric cancer patients enrolled in the National Cancer Institute Cancer Therapy Evaluation Program sponsored phase 1 trials
}

\author{
Hideaki Bando ${ }^{1,2,3}\left(\mathbb{D} \cdot\right.$ Larry Rubinstein $^{4} \cdot$ Pamela Harris $^{1} \cdot$ Takayuki Yoshino $^{2}$ • \\ Toshihiko Doi ${ }^{2} \cdot$ Atsushi Ohtsu ${ }^{2,3} \cdot$ John Welch $^{5} \cdot$ Naoko Takebe $^{1}$
}

Received: 18 March 2016/ Accepted: 2 August 2016/Published online: 10 August 2016

(c) The International Gastric Cancer Association and The Japanese Gastric Cancer Association 2016

\begin{abstract}
Background In phase 1 trials, an important entry criterion is life expectancy predicted to be more than 90 days, which is generally difficult to predict. The Royal Marsden Hospital (RMH) prognostic score that is determined by lactate dehydrogenase level, albumin level, and number of metastatic sites of disease was developed to help project patient outcomes. There have been no systematic analyses to evaluate the utility of the RMH prognostic score for esophagogastric cancer patients.

Methods All nonpediatric phase 1 oncology trials sponsored by the National Cancer Institute Cancer Therapy Evaluation Program that began between 2001 and 2013 were considered in this review.
\end{abstract}

Hideaki Bando

hbando@east.ncc.go.jp

1 Investigational Drug Branch, Cancer Therapy Evaluation Program, Division of Cancer Treatment and Diagnosis, National Cancer Institute, National Institutes of Health, 9609 Medical Center Drive, Bethesda, MD 20892, USA

2 Department of Gastroenterology and Gastrointestinal Oncology, National Cancer Center Hospital East, 6-5-1 Kashiwanoha, Kashiwa, Chiba 277-8577, Japan

3 Course of Advanced Clinical Research of Cancer, Juntendo University Graduate School of Medicine, 2-1-1, Hongo, Bunkyo-ku, Tokyo 113-8421, Japan

4 Biometric Research Program, Division of Cancer Treatment and Diagnosis, National Cancer Institute, National Institutes of Health, 9609 Medical Center Drive, Bethesda, MD 20892, USA

5 Center for Global Health, National Cancer Institute, National Institutes of Health, 9609 Medical Center Drive, Bethesda, MD 20892, USA
Results Of 4722 patients with solid tumors, 115 patients were eligible for our analysis; 54 (47\%) with cancer of the esophagus, $14(12 \%)$ with cancer of the esopagogastric junction, and $47(41 \%)$ with stomach cancer. Eighty-six patients $(75 \%)$ had a good RMH prognostic score $(0$ or 1$)$ and 29 patients $(25 \%)$ had a poor RMH prognostic score (2 or 3). Disease control rates were significantly different between patients with good and poor RMH prognostic scores (49\% vs $17 \%$; two-sided Fisher's exact test $P=0.004)$. The median treatment duration and overall survival for good and poor RMH prognostic score patients were significantly different (median treatment duration 2.1 months vs 1.2 months respectively, $P=0.016$; median overall survival 10.9 months vs 2.1 months respectively, $P<0.001$ ). In the multivariate analysis, age (60 years or older), Eastern Cooperative Oncology Group performance status (2 or greater), and the RMH prognostic score (2 or 3 ) were significant predictors of poor survival.

Conclusions The RMH prognostic score is a strong tool to predict the prognosis of esophagogastric cancer patients who might participate in a phase 1 trial.

Keywords Phase 1 trials - Esophagogastric cancer . National Cancer Institute · Cancer Therapy Evaluation Program · Royal Marsden Hospital prognostic score

\section{Introduction}

Esophageal and gastric cancers are, respectively, the eighth and fifth commonest malignancies in the world [1]. In 2012, an estimated 456,000 new cases of esophageal cancer and 951,000 new cases of esophageal and stomach cancer occurred worldwide. Both diseases often present in advanced stages because of late onset of symptoms and, 
because of the limited availability of effective treatment strategies, are associated with poor survival. There were approximately 400,000 deaths from esophageal cancer and 723,000 deaths from gastric cancer in 2012 [1].

Most esophageal cancers worldwide are squamous cell carcinoma or adenocarcinoma in origin. Although the incidence of squamous cell carcinoma has decreased in the USA, the incidence of adenocarcinoma stemming from Barrett's esophagus has been increasing dramatically [2]. Similarly, the incidences of adenocarcinoma of the esophagogastric junction and proximal part of the stomach have increased, whereas the incidence of distal gastric carcinoma has declined [3].

The incidence of gastric cancer differs with different geographic regions. The rates are highest in eastern Asia, eastern Europe, and South America, and are lowest in North America and parts of Africa [1]. Furthermore, as the worldwide incidence of gastric cancer has declined, especially in North America and western Europe, gastric cancer has become less common in the USA [4].

Patients with unresectable and recurrent esophagogastric cancer (EGC) at time of diagnosis are usually treated with systemic chemotherapy. At present, fluoropyrimidine and platinum-based chemotherapy with or without trastuzumab, a human epidermal growth factor receptor 2 antibody, are globally regarded as standard first-line chemotherapy for esophagogastric adenocarcinoma [5, 6]. Recently, owing to randomized studies, taxanes, irinotecan, and ramucirumab, an antivascular endothelial growth factor receptor 2 antibody, have been regarded as standard second-line therapeutic options [7-11]. However, the prognosis of patients with advanced or recurrent EGC remains poor, with a median overall survival (OS) of only 12 months.

When patients do not respond to conventional systemic chemotherapy but have a good performance status (PS), they are often candidates for clinical trials. Phase 1 trials are designed primarily to evaluate the tolerability and toxicity profile of new therapies and to determine the recommended phase 2 dose. The generally accepted inclusion and exclusion criteria for these trials include adequate organ function and reasonable PS to ensure safety and avoid unnecessary toxicity. Life expectancy predicted to be less than 90 days is also used to exclude patients with poor prognosis although this is notoriously difficult to predict. There have been various analyses to identify the variables to detect poor prognosis. Recently, the Royal Marsden Hospital (RMH) prognosis score was developed to help predict the outcomes of patient in phase 1 trials. From the multivariate analysis of the RMH phase 1 data set, lactate dehydrogenase (LDH) level, albumin level, and number of metastatic sites of disease were selected as significantly poorer prognostic factors [12]. The RMH prognostic score has also been validated with various prospectively and retrospectively selected cohorts [13, 14].

The Cancer Therapy Evaluation Program (CTEP) of the National Cancer Institute (NCI) in the USA coordinates and supports the largest publicly funded oncology clinical trials program in the world. NCI-CTEP is currently supporting 180 phase 1 clinical trials. NCI-CTEP also manages and provides about 100 investigational new drugs for NCI-CTEP-sponsored clinical trials.

There have been few analyses of clinical benefits and prognoses for EGC patients who were registered in phase 1 trials [15, 16]. Those analyses assessed the toxicities, treatment-related trial discontinuations, and efficacies. However, the impact of phase 1 treatment on safety and efficacy for patients with EGC has not yet been evaluated in a large population. Although the prognostic variables for OS have been analyzed [15], the usefulness of the RMH prognostic score for EGC patients has not yet been evaluated.

In this analysis, we retrospectively investigated the characteristics and clinical benefits in patients who participated in NCI-CTEP-sponsored phase 1 clinical trials. We also investigated whether the RMH prognostic score is a useful tool to predict the prognosis of EGC patients who might participate in phase 1 trials.

\section{Methods}

\section{Patient eligibility}

All nonpediatric phase 1 oncology trials sponsored by NCICTEP initiated between 2001 and 2013 involving enrolled patients with EGC were analyzed in this study. These trials were conducted at the National Institutes of Health Clinical Center and other academic institutions in the USA. In this analysis, we excluded phase $1 / 2$ trials.

Data were provided from the Clinical Trials Monitoring System (CTMS) database, which is managed by Theradex Systems (Princeton, NJ, USA). The CTMS database is prospectively maintained, with robust data management and auditing practices [17]. We received the anonymized clinical data, containing the types of trials, patients' characteristics, safety profiles, and clinical efficacies as in previous reports [18].

\section{The Royal Marsden Hospital prognostic score}

The RMH prognostic score was determined by three variables: LDH level, albumin level, and number of metastatic sites of disease. LDH values greater than the upper limit of normal, albumin level less than $3.5 \mathrm{~g} / \mathrm{dl}$, and more than two metastatic sites each received one point. A total score of 0 
or 1 indicates a good prognosis, whereas a total score of 2 or 3 denotes a poor prognosis [12].

\section{End points and statistical methods}

All statistical evaluations were performed by the primary investigator (H.B.) and the statistician (L.R.). Summary statistics of patient characteristics were provided; KaplanMeier product limit estimates were used to generate curves for treatment duration and OS. Treatment duration was defined as the time from the start of phase 1 treatment to the time of discontinuation as judged by the investigator, due to objective or clinical disease progression, intolerable toxicities, or death. OS was defined as the time from the start of phase 1 treatment to death or the last date the patient was known to be alive. For OS, patients were censored at the time of their last follow-up if they were still alive. Responses in phase 1 studies were assessed according to the Response Evaluation Criteria in Solid Tumors version 1.0 or version 1.1 .

Fisher's exact test was used to compare the differences in the toxicities, response rates, and disease control rates among the groups with different RMH prognostic scores. A log-rank test was used to assess the differences in treatment duration and OS among the groups with different RMH prognostic scores. Univariate and multivariate Cox proportional hazards models were fit to test the covariate effect on OS. Age, sex, tumor location, Eastern Cooperative Oncology Group (ECOG) PS, hemoglobin value, platelet count, LDH level, albumin level, number of metastatic sites, RMH prognostic score, and number of previous treatments were included in an initial multivariate Cox proportional hazards model. A forward selection method model was also used. Covariates with $P<0.05$ were retained in the model.

All analyses were done with IBM SPSS Statistics version 21.0 (IBM, Tokyo, Japan). All reported $P$ values were for two-sided tests, and $P<0.05$ was considered statistically significant.

\section{Results}

\section{Patient characteristics}

Data from 186 CTEP phase 1 trials conducted between February 2001 and July 2013 were included in this analysis. A total of 4722 patients were enrolled in these trials, and 126 patients $(2.7 \%)$ had EGC. Eleven cases were omitted from the analyses because insufficient clinical data were available (type of trial, patient characteristics, safety profiles, clinical effects, etc.). Thus, we analyzed 115 patients with EGC from 44 CTEP phase 1 studies.

Eighty-four patients $(73.0 \%)$ were men and the median patient age was 59 years (range 30-85 years). Most patients were Caucasian $(77.4 \% ; n=89)$. Fifty-four cancer cases $(47 \%)$ were located in the esophagus, 14 (12\%) were in the esophagogastric junction, and 47 (41\%) were in the stomach. The commonest pathologic subtype was adenocarcinoma $(89.6 \% ; n=103)$. Twentysix patients $(22.6 \%)$ had an ECOG PS of 0,84 patients $(73.0 \%)$ had an ECOG PS of 1, and 5 patients $(4.3 \%)$ had an ECOG PS of 2. The median number of prior treatments was 4 (range 0-13). Forty-two patients $(36.5 \%)$ had undergone surgery and 31 patients $(27.0 \%)$ had received radiation therapy. Of the 115 eligible patients, 86 patients (75\%) had a good RMH prognostic score and 29 patients (25\%) had a poor RMH prognostic score (Table 1).

\section{Treatments}

Of 44 CTEP phase 1 studies, 17 were with single agents (3 chemotherapy only and 14 biologic agent only) and 27 were with combination therapy (10 biologic combination and 17 biologic agent plus chemotherapy).

Thirty-two (28\%) of the 115 patients were treated with a single agent and $83(72 \%)$ were treated with combination therapy. Of the 32 patients treated with a single agent, $8(7 \%)$ were treated with a cytotoxic agent, and $24(21 \%)$ were treated with a biologic therapy. Of the 83 patients treated with combination therapy, $18(16 \%)$ were treated with biologic agent combination therapy, and $65(56 \%)$ were treated with biologic and cytotoxic agents (Table 2).

\section{Safety}

Among the 115 patients, 87 patients $(75.7 \%)$ experienced grade 3 or 4 adverse events; these events included 29 cases of neutropenia, 13 cases of thrombocytopenia, 12 cases of diarrhea, 10 cases of nausea, and 8 cases of vomiting. However, only one grade 5 event $(0.9 \%)$ was observed in this analysis. When we counted dose-limiting toxicity (DLT) events, 21 cases were observed (ten blood system disorders, five general disorders, five gastrointestinal disorders, and one cardiac disorder) (Table 3).

Although there were trends that adverse events of a higher grade occurred in patients with a poor RMH prognostic score, we did not observe statistical significance either for grade 3 or 4 adverse events (two-sided $P=0.142$ by Fisher's exact test) or for dose-limiting toxicity events (two-sided $P=0.406$ by Fisher's exact test) between patients with a good versus a poor RMH prognostic score (Table 3). 
Table 1 Patient characteristics $(N=115)$

\begin{tabular}{|c|c|}
\hline Characteristics & Values \\
\hline \multicolumn{2}{|l|}{ Age } \\
\hline Median (years) & 59 \\
\hline Range (years) & $30-85$ \\
\hline \multicolumn{2}{|l|}{ Sex } \\
\hline Male & 84 \\
\hline Female & 31 \\
\hline \multicolumn{2}{|l|}{ Ethnicity } \\
\hline Caucasian & 89 \\
\hline Asian & 5 \\
\hline African & 4 \\
\hline Others & 3 \\
\hline Unknown & 14 \\
\hline \multicolumn{2}{|l|}{ Tumor location } \\
\hline Esophagus & 54 \\
\hline EGJ & 14 \\
\hline Stomach & 47 \\
\hline \multicolumn{2}{|l|}{ Histologic type } \\
\hline Adenocarcinoma & 103 \\
\hline Squamous cell carcinoma & 10 \\
\hline Not known & 2 \\
\hline \multicolumn{2}{|l|}{ ECOG PS } \\
\hline 0 & 26 \\
\hline 1 & 84 \\
\hline 2 & 5 \\
\hline Prior surgery & 42 \\
\hline Prior radiation therapy & 31 \\
\hline \multicolumn{2}{|l|}{ No. of metastatic sites } \\
\hline Median & 2 \\
\hline Range & $2-6$ \\
\hline$\leq 2$ & 85 \\
\hline$>2$ & 30 \\
\hline \multicolumn{2}{|l|}{ Hemoglobin level } \\
\hline Median (g/dl) & 11.9 \\
\hline Range (g/dl) & $9.0-16.0$ \\
\hline \multicolumn{2}{|l|}{ LDH level } \\
\hline Median (U/l) & 183 \\
\hline Range (U/l) & $91-3156$ \\
\hline \multicolumn{2}{|l|}{ Platelet count } \\
\hline Median $\left(\times 10^{3} / \mu \mathrm{l}\right)$ & 260 \\
\hline Range $\left(\times 10^{3} / \mu \mathrm{l}\right)$ & $104-797$ \\
\hline \multicolumn{2}{|l|}{ Albumin level } \\
\hline Median (g/dl) & 3.6 \\
\hline Range (g/dl) & $2.1-4.6$ \\
\hline \multicolumn{2}{|l|}{$\mathrm{RMH}$ prognostic score } \\
\hline 0 & 40 \\
\hline 1 & 46 \\
\hline 2 & 25 \\
\hline 3 & 4 \\
\hline
\end{tabular}

Table 1 continued

\begin{tabular}{lc}
\hline Characteristics & Values \\
\hline $\begin{array}{l}\text { No. of previous treatments } \\
0-2\end{array}$ & 44 \\
3 or 4 & 20 \\
$>4$ & 51 \\
\hline$E C J$ esophagogastric junction, ECOG PS Eastern Cooperative \\
Oncology Group performance status, LDH lactate dehydrogenase, \\
$R M H$ Royal Marsden Hospital
\end{tabular}

Table 2 Phase 1 studies $(N=115)$

\begin{tabular}{lc}
\hline Type of phase 1 study & Patients \\
\hline Monotherapy (17 studies) & $32(28 \%)$ \\
Chemotherapy only (3 studies) & $8(7 \%)$ \\
Biologic only (14 studies) & $24(21 \%)$ \\
Combination therapy (27 studies) & $83(72 \%)$ \\
Chemotherapy combination (0 study) & $0(0 \%)$ \\
Biologic combination (10 studies) & $18(16 \%)$ \\
Biologic and chemotherapy (17 studies) & $65(56 \%)$ \\
Total (44 studies) & $115(100 \%)$ \\
\hline
\end{tabular}

\section{Response}

Of the 86 patients with a good RMH prognostic score, 7 partial response cases $(8 \%)$ and 35 stable disease cases (41\%) were reported (response rate $8 \%$, disease control rate $49 \%$ ). On the other hand, of the 29 patients with a poor RMH prognostic score, only one partial response case (3\%) and four stable disease cases $(14 \%)$ were reported (response rate $3 \%$, disease control rate $17 \%$ ). The response rates for patients with good versus poor RMH prognostic scores ( $8 \%$ vs $3 \%$ ) were not significantly different (two-sided $P=0.68$ by Fisher's exact test), whereas the disease control rates for patients with good versus poor RMH prognostic scores (49\% vs $17 \%$ ) were significantly different (two-sided $P=0.004$ by Fisher's exact test) (Table 4).

\section{Treatment duration and overall survival}

The log-rank analysis showed that patients with a good $\mathrm{RMH}$ prognostic score had a median treatment duration of 2.1 months (95\% confidence interval 1.7-2.4 months) compared with those with a poor RMH prognostic score, who had a median treatment duration of 1.2 months $(95 \%$ confidence interval 1.0-1.4), a statistically significant difference $(P=0.016)$ (Fig. 1a).

The median OS of patients with a good RMH prognostic score was 10.9 months $(95 \%$ confidence interval 
Table 3 Safety profiles

\begin{tabular}{lccc}
\hline & $\begin{array}{l}\text { Good RMH } \\
\text { prognostic } \\
\text { score }(0 \text { or } \\
1)(n=86)\end{array}$ & $\begin{array}{l}\text { Poor RMH } \\
\text { prognostic } \\
\text { score }(2 \text { or } \\
\text { 3) }(n=29)\end{array}$ & $\begin{array}{l}\text { Total } \\
(N=115)\end{array}$ \\
\hline Dose limiting toxicity & $14(16 \%)$ & $7(24 \%)$ & $21(18 \%)$ \\
General disorder & $4(5 \%)$ & $1(3 \%)$ & $5(4 \%)$ \\
Gastrointestinal disorder & $3(4 \%)$ & $2(7 \%)$ & $5(4 \%)$ \\
Blood system disorder & $6(7 \%)$ & $4(14 \%)$ & $10(9 \%)$ \\
Cardiac disorder & $1(1 \%)$ & $0(0 \%)$ & $1(1 \%)$ \\
Adverse events of grade 3 & $62(72 \%)$ & $25(86 \%)$ & $87(76 \%)$ \\
$\quad \begin{array}{l}\text { or 4 } \\
\text { Adverse events of grade 5 } \\
\text { (treatment-related death) }\end{array}$ & $0(0 \%)$ & $1(3 \%)$ & $1(1 \%)$ \\
\hline
\end{tabular}

RMH Royal Marsden Hospital

Table 4 Treatment responses $(N=115)$

\begin{tabular}{|c|c|c|c|}
\hline Best response & $\begin{array}{l}\text { Good } \\
\text { RMH } \\
\text { prognostic } \\
\text { score }(0 \text { or } \\
\text { 1) }(n=86)\end{array}$ & $\begin{array}{l}\text { Poor RMH } \\
\text { prognostic } \\
\text { score }(2 \text { or } \\
3) \\
(n=29)\end{array}$ & $\begin{array}{l}\text { Total } \\
(N=115)\end{array}$ \\
\hline Complete response & $0(0 \%)$ & $0(0 \%)$ & $0(0 \%)$ \\
\hline Partial response & $7(8 \%)$ & $1(3 \%)$ & $8(7 \%)$ \\
\hline Stable disease & $35(41 \%)$ & $4(14 \%)$ & $39(34 \%)$ \\
\hline Progressive disease & $30(35 \%)$ & $22(76 \%)$ & $52(45 \%)$ \\
\hline Not evaluated & $14(16 \%)$ & $2(7 \%)$ & $16(14 \%)$ \\
\hline Total & $86(100 \%)$ & $29(100 \%)$ & $\begin{array}{l}115 \\
(100 \%)\end{array}$ \\
\hline $\begin{array}{l}\text { Response rate (complete } \\
\text { response }+ \text { partial } \\
\text { response) }\end{array}$ & $8 \%$ & $3 \%$ & $7 \%$ \\
\hline $\begin{array}{l}\text { Disease control rate } \\
\quad(\text { complete } \\
\text { response }+ \text { partial } \\
\text { response }+ \text { stable disease })\end{array}$ & $49 \%$ & $17 \%$ & $41 \%$ \\
\hline
\end{tabular}

$R M H$ Royal Marsden Hospital

8.1-13.7 months), whereas for those with a poor RMH prognostic score it was 2.1 months (95\% confidence interval 1.3-2.8), which was also a statistically significant difference by the log-rank analysis $(P<0.0001)$ (Fig. 1b).

\section{Prognostic value of the Royal Marsden Hospital prognostic score}

In the univariate Cox proportional hazards model of OS, ECOG PS ( 0 or 1 vs $2 ; P<0.0001)$, LDH level (upper limit of normal or lower vs above the upper limit of normal ; $P<0.0001)$, albumin level $(3.5 \mathrm{~g} / \mathrm{dl}$ or greater vs less than $3.5 \mathrm{~g} / \mathrm{dl} ; P<0.0001$ ), and the RMH prognostic score (poor vs good; $P<0.0001$ ) were significantly associated
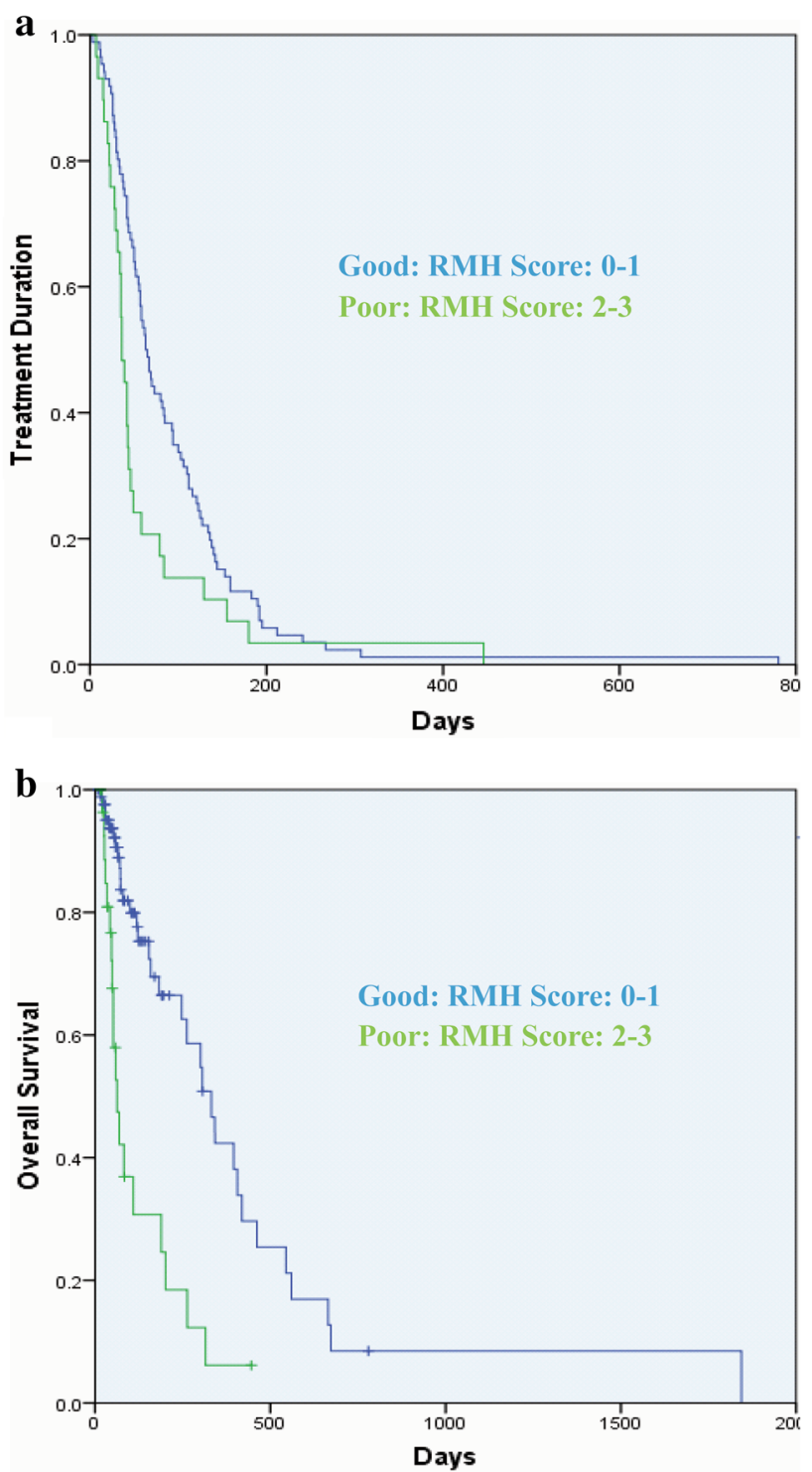

Fig. 1 Kaplan-Meier plots of treatment duration and overall survival for patients with good and poor Royal Marsden Hospital $(R M H)$ prognostic scores. a The median treatment durations of patients with a good RMH prognostic score $(0$ or $1 ; n=86)$ and a poor $\mathrm{RMH}$ prognostic score ( 2 or $3 ; n=29$ ) were respectively 2.1 months [95 \% confidence interval (CI) 1.7-2.4] and 1.2 months (95\% CI 1.0-1.4), which was a statistically significant difference by the log-rank analysis $(P=0.016)$. b The median overall of patients with a good RMH prognostic score was 10.9 months (95\% CI 8.1-13.7 months), whereas for those with a poor RMH prognostic score it was 2.1 months (95\% CI 1.3-2.8), which was also a statistically significant difference by the log-rank analysis $(P<0.0001)$

with a poor phase 1 clinical trial OS (Table 5). In the multivariate analysis with the RMH prognostic score, but not including its components (LDH level, albumin level, and number of metastatic sites of disease), the RMH prognostic score (poor vs good; $P<0.0001$; hazard ratio 0.294 ), age (younger than 60 years vs 60 years or older; $P=0.046$; hazard ratio 0.562$)$, and ECOG PS (0 or 1 vs 2 ; 
Table 5 Univariate and multivariate analysis of overall survival by patient characteristics $(N=115)$

\begin{tabular}{|c|c|c|c|c|}
\hline \multirow[t]{2}{*}{ Characteristics } & \multirow{2}{*}{$\begin{array}{l}\text { Univariate analysis } \\
P\end{array}$} & \multicolumn{3}{|c|}{ Multivariate analysis $^{\mathrm{a}}$} \\
\hline & & Hazard ratio & $95 \% \mathrm{CI}$ & $P$ \\
\hline \multicolumn{5}{|l|}{ Age (years) } \\
\hline $\begin{array}{l}<60(n=58) \\
\geq 60(n=57)\end{array}$ & 0.064 & 0.562 & $0.320-0.990$ & 0.046 \\
\hline \multicolumn{5}{|l|}{ Sex } \\
\hline $\begin{array}{l}\text { Female }(n=31) \\
\text { Male }(n=84)\end{array}$ & 0.475 & & & \\
\hline \multicolumn{5}{|l|}{ Tumor location } \\
\hline $\begin{array}{l}\text { Esophagus, EGJ }(n=68) \\
\text { Stomach }(n=47)\end{array}$ & 0.198 & & & \\
\hline \multicolumn{5}{|l|}{ ECOG PS } \\
\hline $\begin{array}{l}0 \text { or } 1(n=110) \\
2(n=5)\end{array}$ & $<0.001$ & 0.214 & $0.059-0.778$ & 0.019 \\
\hline \multicolumn{5}{|l|}{ Hemoglobin level (g/dl) } \\
\hline $\begin{array}{l}<10(n=12) \\
\geq 10(n=103)\end{array}$ & 0.127 & & & \\
\hline \multicolumn{5}{|l|}{ Platelet count $\left(\times 10^{3} / \mu 1\right)$} \\
\hline $\begin{array}{l}<150(n=12) \\
\geq 150(n=103)\end{array}$ & 0.76 & & & \\
\hline \multicolumn{5}{|l|}{ LDH level } \\
\hline $\begin{array}{l}\leq \mathrm{ULN}(n=73) \\
>\mathrm{ULN}(n=42)\end{array}$ & $<0.001$ & & & \\
\hline \multicolumn{5}{|l|}{ Albumin level (g/dl) } \\
\hline $\begin{array}{l}\geq 3.5(n=79) \\
<3.5(n=36)\end{array}$ & $<0.001$ & & & \\
\hline $\begin{array}{l}\text { No. of metastatic sites } \\
\begin{array}{l}0-2(n=85) \\
>2(n=30)\end{array}\end{array}$ & 0.111 & & & \\
\hline \multicolumn{5}{|l|}{ RMH prognostic score } \\
\hline $\begin{array}{l}0 \text { or } 1(n=86) \\
2 \text { or } 3(n=29)\end{array}$ & $<0.001$ & 0.294 & $0.159-0.545$ & $<0.001$ \\
\hline \multicolumn{5}{|l|}{ No. of previous treatments } \\
\hline $\begin{array}{l}\leq 3(n=56) \\
>3(n=59)\end{array}$ & 0.128 & & & \\
\hline
\end{tabular}

CI confidence interval, ECJ esophagogastric junction, ECOG PS Eastern Cooperative Oncology Group performance status, $L D H$ lactate dehydrogenase, $R M H$ Royal Marsden Hospital, $U L N$ Institutional upper limit of normal

${ }^{a}$ Multivariate analysis with RMH prognostic score but not including its components (LDH level, albumin level, and number of metastatic sites of disease)
$P=0.019 ;$ HR 0.214 ) were significantly associated with a poor OS (Table 5).

\section{Discussion}

We comprehensively reviewed phase 1 oncology trials sponsored by NCI-CTEP between 2001 and 2013 and investigated the characteristics and clinical benefits in enrolled patients with EGC. Of 4722 patients with a solid tumor, we found only 126 patients $(2.7 \%)$ with EGC. This suggests that EGC is a minor component of NCI-CTEPsponsored phase 1 trials in the USA compared with East Asian countries [16].

Because the main NCI-CTEP-sponsored phase 1 trials use combination therapies with investigational new drugs which completed a dose-finding phase 1 trial as a single agent, the combination therapies with biologic agents 
(biologic combination or biologic plus chemotherapy) accounted for the majority (total $72 \%$ ) of phase 1 trials for EGC.

In this analysis, $18.3 \%$ of patients with EGC had doselimiting toxicity events and $75.7 \%$ had grade 3 or 4 adverse events. The $75.7 \%$ of patients with grade 3 or 4 adverse events is considerably higher than reported by previous analyses (19.7-39\%) [15, 16]. A reasonable explanation is that the main NCI-CTEP-sponsored phase 1 trials for EGC were for combinations with biologic and cytotoxic agents. Twenty-seven patients received combinations with cisplatin, irinotecan, and a cyclin-dependent kinase 9 inhibitor and seven received combinations with leucovorin, 5-fluorouracil, and irinotecan (FOLFIRI) and a cyclin-dependent kinase 9 inhibitor. Those treatment regimens are expected to yield a high level of hematologic adverse events. On the other hand, grade 5 adverse events were rare $(0.9 \%)$.

Despite the finding that expected survival of more than 3 months is frequently one of the eligibility criteria for enrollment in most phase 1 trials, clinicians who screen patients often fail to accurately predict individual survival profiles, and as many as 15-20\% of these patients die within the first 3 months of phase 1 trial entry [19]. In our analysis, the median OS of patients with good $\mathrm{RMH}$ prognostic scores and poor RMH prognostic scores were 10.9 and 2.1 months respectively; this was a statistically significant difference in the log-rank analysis $(P<0.0001)$. The median OS of 2.1 months for patients with poor $\mathrm{RMH}$ prognostic scores was shorter than the 3 months major entry criterion. In the multivariate analysis, the RMH prognostic score was also significantly associated with a poor OS. The significant difference in OS between patients with good $\mathrm{RMH}$ prognostic score and patients with a poor RMH prognostic score was also consistent in the separate age groups (younger than 60 years, median OS 9.9 months vs 1.9 months, $P=0.001$; 60 years or older, 11.2 months vs 2.7 months, $P=0.004$ ).

Our analysis has some limitations. We retrospectively used the CTMS database to analyze patient characteristics, treatment duration, and OS since we could not access the medical records of each patient. Since most NCI-CTEPsponsored phase 1 clinical trials do not consistently capture the date of death when patients are no longer included in the study, this information was captured for only 52 patients $(45 \%)$. Since the patients with good RMH prognostic scores tended to live longer than those with poor RMH prognostic scores, the date of death was captured less frequently for the former $(41 \%)$ than for the latter $(66 \%)$. Although it is possible that this introduced statistical bias into the calculations of the predictive value of the RMH prognostic score for OS, it is very unlikely that it significantly affected the results.
In conclusion, the RMH prognostic score could be a strong tool to predict the prognosis of EGC patients who will participate in a phase 1 trial. The median OS of 2.1 months for EGC patients with a poor RMH prognostic score also suggests that these patients should not be included in phase 1 clinical trials.

Acknowledgments We thank all of the staff at the National Cancer Institute who supported our study. We also thank Matthew Rinker at Theradex Systems for helping us to arrange the data for analysis.

\section{Compliance with ethical standards}

Funding No grant and contract funds were provided.

Conflict of interest The authors have declared no conflicts of interest.

Ethical standards All procedures followed were in accordance with the ethical standards of the responsible committee on human experimentation (institutional and national) and with the Helsinki Declaration of 1964 and later versions. We analyzed the delinked anonymous data extracted from the Clinical Trials Monitoring Branch database, which is prospectively managed and closely audited by Theradex Systems (Princeton, NJ, USA) in collaboration with National Cancer Institute. Hideaki Bando received ethical training managed by the National Cancer Institute before accessing the database.

\section{References}

1. Ferlay J, et al. Cancer incidence and mortality worldwide: sources, methods and major patterns in GLOBOCAN 2012. Int J Cancer. 2015;136(5):E359-86.

2. Pohl H, Sirovich B, Welch HG. Esophageal adenocarcinoma incidence: are we reaching the peak? Cancer Epidemiol Biomark Prev. 2010;19(6):1468-70.

3. Salvon-Harman JC, et al. Shifting proportions of gastric adenocarcinomas. Arch Surg. 1994;129(4):381-8; discussion 388-9.

4. Siegel R, Naishadham D, Jemal A. Cancer statistics, 2013. CA Cancer J Clin. 2013;63(1):11-30.

5. Bang YJ, et al. Trastuzumab in combination with chemotherapy versus chemotherapy alone for treatment of HER2-positive advanced gastric or gastro-oesophageal junction cancer (ToGA): a phase 3, open-label, randomised controlled trial. Lancet. 2010;376(9742):687-97.

6. Kang YK, et al. Capecitabine/cisplatin versus 5-fluorouracil/cisplatin as first-line therapy in patients with advanced gastric cancer: a randomised phase III noninferiority trial. Ann Oncol. 2009;20(4):666-73.

7. Kang JH, et al. Salvage chemotherapy for pretreated gastric cancer: a randomized phase III trial comparing chemotherapy plus best supportive care with best supportive care alone. J Clin Oncol. 2012;30(13):1513-8.

8. Fuchs CS, et al. Ramucirumab monotherapy for previously treated advanced gastric or gastro-oesophageal junction adenocarcinoma (REGARD): an international, randomised, multicentre, placebo-controlled, phase 3 trial. Lancet. 2014;383(9911): 31-9.

9. Wilke $\mathrm{H}$, et al. Ramucirumab plus paclitaxel versus placebo plus paclitaxel in patients with previously treated advanced gastric or gastro-oesophageal junction adenocarcinoma (RAINBOW): a 
double-blind, randomised phase 3 trial. Lancet Oncol. 2014;15(11):1224-35.

10. Ford HE, et al. Docetaxel versus active symptom control for refractory oesophagogastric adenocarcinoma (COUGAR-02): an open-label, phase 3 randomised controlled trial. Lancet Oncol. 2014;15(1):78-86.

11. Thuss-Patience PC, et al. Survival advantage for irinotecan versus best supportive care as second-line chemotherapy in gastric cancer-a randomised phase III study of the Arbeitsgemeinschaft Internistische Onkologie (AIO). Eur J Cancer. 2011;47(15): 2306-14.

12. Arkenau HT, et al. Clinical outcome and prognostic factors for patients treated within the context of a phase I study: the Royal Marsden Hospital experience. Br J Cancer. 2008;98(6):1029-33.

13. Arkenau HT, et al. Prospective validation of a prognostic score to improve patient selection for oncology phase I trials. J Clin Oncol. 2009;27(16):2692-6.

14. Garrido-Laguna I, et al. Validation of the Royal Marsden Hospital prognostic score in patients treated in the Phase I
Clinical Trials Program at the MD Anderson Cancer Center. Cancer. 2012;118(5):1422-8.

15. Khan K, et al. Phase I trials in patients with relapsed, advanced upper gastrointestinal carcinomas: experience in a specialist unit. Gastric Cancer. 2014;17(4):621-9.

16. Kawazoe A, et al. Clinical outcomes in 66 patients with advanced gastric cancer treated in phase I trials: the NCCHE experience. Invest New Drugs. 2015;33(3):664-70.

17. Ansher SS, Scharf R. The Cancer Therapy Evaluation Program (CTEP) at the National Cancer Institute: industry collaborations in new agent development. Ann N Y Acad Sci. 2001;949:333-40.

18. Hyman DM, et al. Nomogram to predict cycle-one serious drugrelated toxicity in phase I oncology trials. J Clin Oncol. 2014;32(6):519-26.

19. Arkenau HT, et al. 90-Days mortality rate in patients treated within the context of a phase-I trial: how should we identify patients who should not go on trial? Eur $\mathrm{J}$ Cancer. 2008;44(11):1536-40. 